W stużbie tradycji i odnowy liturgicznej. 50 lat Instytutu Liturgicznego w Krakowie (1968-2018), red. P. Nowakowski, J. Mieczkowski, Kraków 2019, s. 113-122.

ISBN 978-83-7438-849-8 (wersja drukowana), ISBN 978-83-7438-850-4 (wersja online)

DOI:http://dx.doi.org/10.15633/9788374388504.10

Giuseppe Guerra CM

REDAKTOR „EPHEMERIDES LiturgicaE”, RZYM, WŁochy

\title{
L'Accademia Liturgica Romana (1840-1970)
}

I prodromi si fanno risalire a Benedetto XIII con il Concilio Romano del 1725 che promosse iniziative formative verso una cultura liturgica; un'attività in tal senso fu svolta dai Pii Operai alla Madonna dei Monti. Tale attività fu costituita come "Accademia Liturgica" da Papa Benedetto XIV con il Postquam intelleximus ( 1 luglio 1748) ed ebbe 10 anni di attività; infatti nel 1758, con la morte del Papa, essa restò inattiva ${ }^{1}$.

Si riprese 82 anni dopo: nel 1840 nella Casa dei Missionari di S. Vincenzo De Paoli, a Montecitorio, che rilanciarono, con la benedizione e l'approvazione di Papa Gregorio XVI, la vita dell'Accademia, forti della loro esperienza delle Conferenze ai Sacerdoti che, continuando l'uso del loro Fondatore, essi radunavano il martedì ${ }^{2}$. Il Fondatore, comunque, resta Benedetto XIV. L'Annuario Pontificio che nell'annata 1912 cita tra le Isti-

I G. Moroni, Dizionario di erudizione storico-ecclesiastica: da S. Pietro ai nostri giorni specialmente intorno ai principali santi, beati, martiri, padri, ai sommi pontefici, cardinali e più celebri scrittori..., Venezia 1840; G. B. Angnolucci C.M., L'Accademia Liturgica romana, Roma 1931, p. 25 (l'autore descrive questo periodo, attingendo ai "Spunti" stampati dall'Accademia).

2 Che Benedetto XIV abbia fondato l'Accademia Liturgica, affidandola ai Missionari (quell' Accademia che è durata poi fino ai giorni nostri), è un'affermazione generica che ingloba varie epoche e sviluppi graduali che vanno precisati. 
tuzioni culturali l'Accademia Liturgica Romana, nell'edizione del 1937, p. 822 precisa la data 1740 e il Fondatore Benedetto XIV; nel 1944, p. 810 aggiunge "ricostituita da Gregorio XVI nel 1840".

Possiamo dire che l'Accademia Liturgica, animata dai Missionari di S. Vincenzo, ha mantenuto sia lo spirito sia la struttura fondamentale per più di 130 anni, fino agli anni del Concilio Vaticano II e del post-concilio, possiamo dire fino al 1970, dopo del quale ovviamente, se non vogliamo dire che è finita, si deve dire che ha subito una profonda metamorfosi.

Nell'Annuario Pontificio 1'Accademia Liturgica viene citata presso la Casa dei Missionari Vincenziani fino al $1969^{3}$ (anche se poi continua a citarla per inerzia fino al 1993). Il nome del direttore nell'Annuario Pontificio inizia a essere indicato (con P. Angelo Martorelli C.M.) dal 1917 fino al 1969 (Direttore P. Luigi Pizzoni).

Direttori e Padri della Congregazione della Missione hanno scritto celebri manuali come: Felice Zualdi ${ }^{4}$, Pietro Maria De Amicis ${ }^{5}$, P. Luigi Iamarco $^{6}$, P. Francesco Stella7.

\section{Dal 1840 fino agli anni ‘ 30.}

Questo periodo, dalla rifondazione del 1840 al Concilio Vaticano II si può descrivere con la documentazione dell' Archivio dell' Accademia, già utilizzata dall' Angioletti nel suo scritto: Giovanni Battista Angnolucci C.M., L'Accademia Liturgica romana, pubblicato come Estratto (ma è piuttosto un Supplemento) nel 1931.

3 "Annuario Pontificio" 1912, p. 580: Accademia Liturgica a Montecitorio; "Annuario Pontificio" del 1937 sulla p. 822 precisa: Fondata nel 1740 da Benedetto XIV; "Annuario Pontificio" nel 1944 sulla p. 810 aggiunge: ricostituita da Gregorio XVI nel 1840.

4 F. Zualdi, Prete della Missione Direttore dell'Accademia Liturgica di Roma, Cerimonie della Messa privata, Bologna 1884 .

5 Istruzioni, Ceremonie e Riti delle Sacre Ordinazioni, Roma 1901; Caeremoniale Parochorm in due volumi, Roma 1910.

6 Sacre Cerimonie per la Messa solenne, Napoli 1932.

7 Institutiones Liturgicae in Seminariorum usum, Ephem. Liturg., Libreria vaticana, Roma 1929. 
Le riunioni erano fissate al martedi ${ }^{8}$, il giorno tradizionale delle Conferenze ai Sacerdoti, con una cadenza quindicinale (una quindicina di riunioni da metà novembre a metà agosto) ${ }^{9}$. Si cominciò con lunghe Dissertationes, a volte pubblicate, ma a cura dell'autore (abbiamo per es. una del 25 dicembre 1841, a Natale!). Ma con il Card. Vicario (Card. Naro Patrizi), riprendendo e collegandosi alle disposizioni del Sinodo Romano di Benedetto XIII sui casi di morale e di liturgia (vedi l'Unione di San Paolo Apostolo), fu messa sotto la giurisdizione del Vicariato l'Accademia Liturgica. Uno Statuto e un Regolamento adeguato alla nuova impostazione furono stampati nel 1854 .

Si diede una impronta più pratica, come si vede dalle Quaestiones liturgicae o Rituales pubblicate. Tutte le Quaestiones furono stampate, dal 1840 in poi $^{10}$. Le Solutiones, affidate agli iscritti e ai frequentatori dell' Accademia, sono stampate solo in parte. Dal 1927 sono stati conservati solo i manoscritti. Alcune Questioni (le prime tre del 1855 e la quarta del 1856) furono tradotte e pubblicate in francese (Bruxelles, 1859 e 1861).

Nei volumi stampati sono riportati i nomi di quelli che ricoprivano le cariche direttive dell' Accademia (Presidenti, Segretari, Censori) e i membri dell' Accademia elevati alla Sacra Porpora come Cardinali. Per esempio: G. B. Nasalli Rocca, Alfredo Ildebrando Schuster, Domenico Tardini, Gaetano De Lai, Pietro La Fontaine.

Nell'Archivio dell' Accademia conserviamo i Registri dei frequentatori dell'Accademia: Catalogo dei Sig.i Ecclesiastici che frequentano l'Accademia Liturgica 1879-1913; 1913-1939; 1939-1964.

Alla fine dell'anno vi erano Premiazione, Certificati. Come per le Iscrizioni all' Accademia e per l'elenco dei Responsabili (Segretari, Sottosegretari, Censori ecc.) e per le rispettive nomine secondo le regole dello Statuto, tutti gli Atti e i Verbali sono riportati nell' Archivio dell'Accademia.

La partecipazione all'Accademia diveniva un titolo quasi indispensabile per gli Uffici liturgici della Curia. Nel Verbale del Consiglio dell' Accademia del 20 ottobre 1955, ci si scandalizza del fatto riportato dall' “Osservatore Romano" del 2 agosto 1955: sono stati nominati Cerimonieri Pontifici persone

8 Poi si cambiò al mercoledì.

9 Negli anni 6o cadenza quasi sempre mensile (non più quindicinale).

10 Non quelle dal 1847 al 50 . Non quelle dal 1857 all' 83 . 
che non sono appartenute all' Accademia Liturgica ${ }^{11}$. Era una disposizione della Segreteria di Stato del 1879, ma pare che durò fino al $1946^{12}$.

L'Elenco dei Risolventi l'abbiamo in un Index di "Ephemerides Liturgicae" 1887-1926. Riportati in un manoscritto quelli dal 1840 al 1843, che altrimenti non ci sarebbero notati in quanto i nomi non furono riportati nelle stampe.

Dopo le Solutiones, c'era un'esortazione del "Missionario Vincenziano" (alcune sono state conservate). E nelle Feste di S. Vincenzo e dell' Assunta (poi Immacolata) veniva invitato un oratore.

Nel 1918 su San Vincenzo e nel 1920 sull'Immacolata ha parlato Giovanni Battista Nasalli Rocca che sarebbe divenuto nel 1921 Arcivescovo di Bologna (pubblicato in un estratto di Verbum Domini).

Nel 1923-1924 ha parlato sull'Immacolata Angelo Roncalli' ${ }^{13}$ (era Presidente del Consiglio centrale della Pia Opera di Propaganda Fide). Sacri Oratores in Academia Liturgica in Ephem. Lit. 1931, 7-8.

Il Discorso su S. Vincenzo fu tenuto nel 1921 da Alfredo Idelfonso Schuster, che era divenuto Abate di San Paolo fuori le mura 3 anni prima a soli 38 anni; nel 1922 da Mons. Domenico Tardini (aveva 10 anni di Messa) ${ }^{14}$; e nel $1938^{15}$ da Alfredo Ottaviani che era allora Assessore al S. Uffizio. Il Secondo centenario della canonizzazione di S. Vincenzo (1938), con la celebrazione del 22 aprile $1938^{16} \mathrm{fu}$ anche l'occasione di una ricostruzione storica dell' Accademia Liturgica illustrata dal Direttore dell' Accademia P. Luigi Paladini ${ }^{17}$.

I Casi presentati erano presentati nel Bollettino del Clero Romano (vedi 19 (1938) 50-61, dove fu pure riportata la Giornata Vincenziana del Clero

11 Furono inseriti nell'Accademia post factum (cf. verbale del 25 ottobre 1956).

12 Pro-memoria di P. Giuseppe Pizzoni del 3 aprile 1964.

13 Nel Giornale dell'Anima, Roma 1964, p. 204. Agli anni: 1921-1924, scrive: Esercizi Spirituali, Roma, Villa Carpegna 13-19 gennaio 1924: Interverrò fedelmente, almeno, al caso morale; se posso al liturgico (CJC 131).

14 Per impegni, invece, si scusò per non poter partecipare alla Celebrazione del bicentenario della canonizzazione di S. Vincenzo il 21 aprile 1938.

15 Il Discorso fu pubblicato nell'“Osservatore Romano” del 30 aprile 1938.

16 "Bollettino del Clero romano" 19 (1938), p. 59-61.

17 "Osservatore Romano" del 23 aprile 1938. 
(21 aprile 1937) in vista del $2^{\circ}$ Centenario della canonizzazione di S. Vincenzo (16 giugno 1737-1937)).

Quando i Missionari dovettero lasciare Montecitorio nel $1913^{18}$, cioè anche quella parte della Casa che era loro rimasta dopo l'incameramento dei beni, si stabilirono all'Apollinare (odierna Università di Santa Croce), l'Accademia ebbe la sua sede all' Apollinare ${ }^{19}$. Quando dovettero lasciare anche l'Apollinare trasferendosi al Leoniano (1920), il Papa Benedetto XV con lettera del 27 dicembre 1920 stabilì che le riunioni dell'Accademia sarebbero continuate all' Apollinare. E così fu fino al $1935^{20}$.

Anche nella preparazione del Codice di diritto Canonico (1918) l'Accademia fu invitata a contribuire, nella persona del Direttore P. Calcedonio Mancini che era Presidente della Commissione Liturgica, ai Canoni riguardanti il Culto Divino (cf. lettera del Card. Pietro Gasparri del 14 gennaio 1910).

\section{2. "Ephemerides Liturgicae" (1887) e il contributo dell'Accademia sulle Riforme di Pio X, di Pio XII e del Concilio Vaticano II}

La Rivista "Ephemerides Liturgicae" fondata da P. Calcedonio Mancini (uno dei grandi liturgisti di Leone XIII) ${ }^{21}$ nel 1887 per divenire l'organo dell'Accademia ${ }^{22}$, lungo gli anni ne è divenuta il riflesso esterno al di là della

\footnotetext{
18 G. Guerra C.M., Roma, Sulle orme dei primi Missionari Vincenziani, “Informazione Vincenziana" 18 (2016), p. 22-25.

19 Vedi "Annuario Pontificio" 1916, p. 602.

20 Cf. G. B. Angnolucci C.M., L'Accademia Liturgica romana, p. 25. L'“Annuario Pontificio" che nel 1935, p. 800 indica Piazza dell'Apollinare, 49, nel 1936 indica Via Pompeo Magno, 21. Nel periodo, poi, in cui l'Accademia e le "Ephemerides" furono stabilite a S. Silvestro (1938-1948) tutto si svolse a S. Silvestro (vedi Verbali 1938-1948). Anche le Riunioni (infatti, quando nel 48 verrà invocata la permanenza a S. Silvestro, si giustificherà con la centralità di S. Silvestro).

21 Necrologio di P. C. Mancini scritto da Pietro Piacenza in "Ephemerides Liturgicae" 24 (1910), p. 513-516. Anche "Ephemerides Liturgicae" 25 (1911), p. 5-6.

22 Appunti manoscritti di una trasmissione alla Radio Vaticana 1961-1962. "La più antica rivista liturgica esistente" la dice P. Annibale Bugnini in Memorie autobiografiche, Roma 2012, p. 36.
} 
città di Roma, ereditandone dopo gli anni del Concilio e del postconcilio la missione e la funzione. Dagli anni ' 40 la Redazione si fece editrice di molte pubblicazioni (attività che continua fino ai nostri giorni) con la collana Bibliotheca Ephemerides Liturgicae, la cui perla, dice P. Bugninii ${ }^{23}$, è costituita dalla Miscellanea Mohlberg, in due volumi. Si aggiunse anche la Collana ${ }^{24}$ Ardens et Lucens.

Il 1887 aveva segnato un importante passo avanti, con il primo numero di "Ephemerides Liturgicae", che divenne l'organo ufficiale ${ }^{25}$ e riportò per tutti gli anni avvenire l'elenco delle questioni che anno per anno venivano proposte nell'Accademia. Molti articoli della Rivista provenivano dai membri dell'Accademia. Ebbe un Regolamento del 1901 approvato dal P. Generale Antonio Fiat. Diveniva un'opera sponsorizzata dalla Congregazione della Missione.

Fino al 1945 i Direttori dell' Accademia (Calcedonio Mancini2 ${ }^{26} 1883-1910$ (Mons. Piacenza 1903-1919)), D'Isegnard Giuseppe 1910-1913, Martorelli Angelo 1913-1920 (1913-1920 siamo all'Apollinare), Marina Giuseppe ${ }^{27}$ 1920-1921, Paladini Luigii ${ }^{28}$ 1921-1943 (1931-1933 Pizzoni, 1938 S. Silvestro), Pizzoni Giuseppe ${ }^{29}$ 1943-1969 (1947 al Leoniano), vengono indicati anonimamente come Presbiteri CM 1887-1945 e fino al 1954 come Coetus (Redazione). P. Annibale Bugnini compare come Segretario nel 1945 e come Direttore dal 1955.

La Rivista uscì mensilmente fino al 1927. Come "Nuova Serie" dal 1927 al 1955 trimestrale ${ }^{30}$. Dal 1956 al 2000 bimestrale. Dal 2001 ad oggi trimestrale.

\footnotetext{
23 A. Bugnini, Memorie autobiografiche, Roma 2012, p. 49. Bugnini in occasione del suo $70^{\circ}$ compleanno, Roma 1982, p. 15 . del 29 agosto 1948 .

$26 \quad 1843-1910$.

27 1887-1950. Ordinato sacerdote 1910, Vescovo 1936.

28 1877-1961.

29 1899-1972.

30 L'annata 1946 fu semestrale.
}

24 Liturgia opera divina e umana, Studi sulla riforma liturgica offerti a S.E. Mons. Annibale

25 G. B. Angnolucci C.M., L'Accademia Liturgica romana, p. 24: “il rev. Sig. Mancini incominciò la pubblicazione del periodico Ephenerides Liturgicae, che divenne l'organo ufficiale dell'Accademia per la pubblicazione delle soluzioni". Vedi anche un appunto di P. A. Bugnini 
Nel 1920 ci fu un rilancio e rinnovamento dell' Accademia ${ }^{31}$, proprio attraverso la "Rivista Ephemerides Liturgicae" ecc. Fu in quegli anni, precisamente 1928-1930, che P. Annibale Bugnini ${ }^{32}$, giovane novizio al Leoniano, trovò "l'inizio della sua vocazione liturgica" 33 ; lì cominciò ad apprezzare nella sua profondità la famosa frase di S. Vincenzo nella Conferenza ai Missionari (n. 141, sett. 1655, ed. Coste 1924, XI, p. 312; trad. italiana, Roma 2008, vol. X, p. 253): “Le cerimonie, è vero, sono solo l'ombra, ma l'ombra delle più grandi cose" ${ }^{\prime 34}$.

Fino al 1922 "la rivista svolse un programma quasi strettamente giuridico-rubrica. Il Direttore P. Luigi Paladini, uomo di cultura e di studio, ma quasi improvvisato in materia" 35 .

$\mathrm{Nel}$ 1937, con la decisione di dividere in due parti, di cui la seconda era intitolata: Jus et Praxis, si cercò di conciliare l'aspetto più storico, scientifico, accademico con quello pastorale e pratico (fu riunificata nel $1948^{36}$ ).

La Rivista dal 1938 fu portata a San Silvestro37, (Via XXIV Maggio). Non era semplicemente un trasferimento logistico; intendeva costituire un gruppo di redattori riuniti in una comunità specificamente impegnata nella liturgia. Dopo l’Enciclica "Mediator Dei" del 22 novembre 1947, P. Annibale Bugnini Direttore della Rivista "Ephemerides Liturgicae", fu nominato membro, anzi Segretario della Commissione "Piana" per la Riforma (28 maggio 1948). La Rivista in quegli anni era "ritenuta voce ufficiosa degli ambienti liturgici romani" ${ }^{38}$. E divenne promotrice di una liturgia

31 Ad opera di P. Alcide Marina C.M., cf. P. A. Bugnini, Memorie autobiografiche, Roma 2012, p. 36 .

32 1912-1982. Consacrato Vescovo nel 1972.

33 A. Bugnini, Memorie autobiografiche, p. 36.

34 A. Bugnini, La riforma liturgica (1948-1975), Roma 1997, p. 21.

35 A. Bugnini, Memorie autobiografiche, p. 36.

36 Consiglio di "Ephemerides Liturgicae" 4 novembre 1947 (Direttore P. A. Bugnini).

37 S. Silvestro era stata la sede di un primissimo studio della liturgia e di attuazione del Concilio di Trento. A. Bugnini, La riforma liturgica (1948-1975), Roma 1997, p. 11.

38 A. Bugnini, La riforma liturgica (1948-1975), Roma 1997, p. 26. In un Pro-memoria del 28 marzo 1948 scrive: “Oggi Ephemerides Liturgicae è una rivista internazionale. Unica nel suo genere dopo la cessazione di Jahrbuch für Liturgiewissenshaft". 
più collegata con la vita pastorale e partecipatrice 39 . "Da una impostazione prevalentemente rubricale e giuridica passò al piano scientifico e pastorale, divenendo strumento di comunicazione di studi e anche di fermenti, aspettative, desideri della Chiesa" ${ }^{40}$.

Ma proprio il 1948 fu l'anno che P. Annibale Bugnini, Direttore della Rivista e dell' Accademia fin dal 1943 in un suo pro-memoria definisce "un terremoto". Il Superiore Provinciale, P. Ernesto Cassinari C.M., volle riportare l'Amministrazione al Leoniano. In conclusione da quell'anno iniziò una certa divisione tra "Ephemerides Liturgicae" (a S. Silvestro) che costituiva il rinnovamento e l'Accademia Liturgica che continuò nelle forme più tradizionali al Leoniano.

P. Annibale Bugnini che da S. Silvestro dovette in quell'anno trasferirsi al Leoniano (dove riprese dal 1955 al 1957 la Direzione del Convitto dei Sacerdoti Studenti che aveva già tenuta dal 1939 al 1943), continuò comunque l'opera di rinnovamento, soprattutto quando ritornò nel 1958 (questa volta come superiore) a S. Silvestro. Più volte ha incoraggiato iniziative di collaborazione con S. Anselmo, con la Gregoriana, con il C.A.L.

Il 14 marzo 1951 Ferdinando Antonelli OFM Relatore generale della Sez. Storica della S. Congregazione dei Riti, tiene una Conferenza su "Il ripristino della solenne veglia pasquale".

Dopo il famoso convegno di Assisi del 1956, l'Accademia si fece promotrice della Settimana pastorale (7-14 luglio 1957) ${ }^{41}$.

Ormai lo spirito e la storia dell' Accademia veniva riassorbita sotto la Direzione del Direttore della Rivista (fino al 1963, Bugnini; poi quando dovette impegnarsi in pieno come Segretario della Commissione Preparatoria del Concilio Vaticano II (nomina che fu la premessa degli ulteriori incarichi che ebbe rendendolo protagonista della riforma liturgica) gli successe il

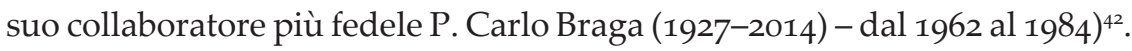

39 D'altra parte nel suo Diario P. A. Bugnini aveva osservato che la formazione nei seminari era accurata, ma l'aspetto umano era carente: “Si cresceva su, come fanciulline di collegio, in una visione irreale, e talvolta falsata della vita". A. Bugnini, Memorie autobiografiche, p. 35 .

40 G. Pasqualetti in: Liturgia opera divina e umana, p. 15.

41 A. Bugnini, Memorie autobiografiche, p. 6o.

42 Poi Pistoia Alessandro (1936-2005) dal 1985 al 2005; e Gioia Eugenio dal 2006 al 2015. 
Nella sua opera (pubblicata postuma) "La riforma liturgica" (1948$1975)^{43}$, P. A. Bugnini cita la Rivista "Ephemerides Liturgicae" come l'espressione del movimento romano verso la riforma liturgica e ricorda gli anni in cui, come Direttore per 20 anni (1943-1963), abbia in essa pubblicato tanti contributi in preparazione al Concilio Vaticano II.

Uno Statuto fu stilato e approvato dal Superiore Provinciale P. Luigi Betta il 15 novembre 1956 (alcune aggiunte, ma non sostanziali, furono apportate nel 1963 dal Superiore Provinciale P. Luigi Franci). Lo Statuto, intitolato E.L.E.V. (Edizioni Liturgiche e Vincenziane) comprendeva sia la Rivista "Ephemerides Liturgicae", sia le altre attività.

L'Accademia ufficialmente e con i suoi raduni settimanali dei "casi" continuava, e continuò fino al 1969.

Nel 1969 (cf. Statuto 21.02.1969) il C.L.V. (Centro Liturgico Vincenziano) comprende tutto, "Ephemerides Liturgicae" ecc. (II Sezione di CLV) e anche E.L.E.V. (III Sezione). Nel $1969 \mathrm{fu}$ organizzato il Corso di Pastorale liturgica per il clero addetto al ministero pastorale; inaugurato il 14 gennaio $1969^{44}$ alla presenza di Mons. Giovanni Canestri Vescovo Ausiliare e presidente della Commissione liturgica Diocesana, proseguì ogni martedì fino all' 11 marzo ${ }^{45}$, avente come tema il Direttorio liturgico-pastorale per l'uso del rituale dei sacramenti e dei sacramentali pubblicato dalla C.E.I. nel giugno del 1967.

Con una parentesi e con un testo (1 marzo 1974 che parla di ELEV che comprende il CLV) arriviamo ai giorni nostri con 31 gennaio 1978: atto Notarile Centro Liturgico Vincenziano.

\section{Streszczenie}

\section{Akademia Liturgiczna w Rzymie od 1840 do 1970 roku}

Wykład przedstawia rozwój historyczny Rzymskiej Akademii Liturgicznej (Accademia Liturgica Romana). Uczelnia ta została przekazana zgromadzeniu

A. Bugnini, La riforma liturgica (1948-1975), 2 ed., Roma 1983.

"Osservatore Romano", 20-21 gennaio 1969.

“Osservatore Romano", 13-14 gennaio 1969. 
misjonarzy w 1840 roku i przez ponad sto lat stanowiła centrum studiów i formacji liturgicznej w Rzymie. Gruntownie zreformowana w czasach Soboru Watykańskiego II, aktualnie nie istnieje, ale za jej owoc może być uznane Liturgiczne Centrum Wincentyńskie (Centro Liturgico Vincenziano) w Rzymie oraz międzynarodowy periodyk „Ephemerides Liturgicae”. 